\title{
CYANOSIS - PART 2 CONTINUATION OF CYANOSIS PART 1
}

\author{
Maddury Jyotsna, B. Srinivas, Nemani Lalita
}

\section{IMPLICATIONS OF CYANOSIS IN A NEONATE:}

1. Coarctation - in a neonate with this condition, one must not try to achieve complete abolition of cyanosis and a saturation of 75 to 85 percent can be adequate to avoid tissue hypoxemia and eventual lactic acidosis.

2. Severe ebstein's anomaly - Usually surgical management strategies are based on tricuspid valve regurgitation and degree of cyanosis. If cyanosis alone is the dominant symptom, a systemic to pulmonary artery shunt alone is performed.

3. Total anomalous pulmonary venous connection and prostaglandin infusion - Routinely, cyanotic CHDs with severe hypoxemia can be put on prostaglandin infusion without any harm before sending them to a tertiary cardiac care center, when the facility for echocardiography is not available and exact diagnosis cannot be made. However, there are some concerns regarding its use in total anomalous pulmonary venous connection (TAPVC) especially the obstructed type. PGE1 could produce adverse effects by two mechanisms: - By increasing the cyanosis and hypoxemia of systemic circulation: When the pulmonary arterial pressure is higher than that in the aorta, dilatation of the DA may result in an increase in right-to-left shunt and would increase in the degree of cyanosis.

- By direct pulmonary vasodilatation leading to altered Qp/Qs. usually, it might be expected that the ductus response to PGE1 would predominate and cyanosis may be expected to worsen.

\section{IMPLICATION OF CYANOSIS IN A CHILD/ADULT:}

Cyanosis in VSD: Combination of VSD, pulmonary vascular disease and cyanosis has been termed as,

Maddury Jyotsna ${ }^{1}$, B. Srinivas ${ }^{2}$, Nemani Lalita ${ }^{2}$,

${ }^{1}$ Professor \& HOU-IV, Department of Cardiology, NIMS, India

${ }^{2}$ Associate Professor, Department of Cardiology, NIMS.

Corresponding Author: Maddury Jyotsna

Email: mail2jyotsna@rediffmail.com
'Eisenmenger complex'. Pulmonary vascular disease and cyanosis in combination with any other systemic-topulmonary connection has been called Eisenmenger syndrome. Despite similar cyanosis, patients with Eisenmenger syndrome show less exercise performance, more ventilation-perfusion mismatch and a worse quality of life when compared to complex cyanotic CHD patients with pulmonary stenosis who are protected from severe pulmonary vascular disease.

Initially cyanosis is internment only during exercise, due to the fall in SVR. Later, the right to left shunt occurs at rest with persistent cyanosis. Infants with nonrestrictive VSDs with balanced shunts may become cyanotic on crying or exercise.VSD with later development of RVOT develops cyanosis.

Cyanosis in PDA: Eisenmenger Syndrome. Characteristic feature is the presence of Differential cyanosis occurs (pink fingers, blue toes).In a large PDA with mild cyanosis in a child, which previously was considered as inoperable or percutaneous closure was considered as contraindicated, now can be tested with temporary occlusion of PDA with balloon and then calculated the cardiac output and measure the right a trial pressure and tolerance of the patient can be tested. Extrapolation of these criteria to the older adult with RV dysfunction and cyanosis is unsubstantiated, but serves as a guide for at least acute testing of physiologic tolerance.

Cyanosis in ASD: Eisenmenger syndrome. Cyanosis is secondary to direction of deoxygenated blood from the IVC through prominent Eustachian valve or the besian valve through the defect to the left atrium or the presence of Common atrium/ TAPVC or PAPVC.

Cyanosis in AV canal defects: Right ventricle-left atrial shunt could be a cause of cyanosis in a child with partial AV canal defect with normal pulmonary artery pressure. A patient of complete AVSD with normal venous drainage and with cyanosis and clubbing in the absence of right ventricular outflow tract obstruction is clearly inoperable. 
Cyanosis in tricuspid atresia: This is the most common cause of cyanosis with left ventricular hypertrophy. An infant with markedly decreased pulmonary blood flow will present early in the neonatal period with severe cyanosis, hypoxemia and acidosis. An infant with markedly increased pulmonary flow does not have significant cyanosis, but usually presents with signs of heart failure. Patients with pulmonary atresia (Subgroup a) irrespective of the type will also present with early cyanosis, especially when the ductus begins to close.

Cyanosis in Ebstein's anomaly: Characterized by 'Transient' or 'intermittent' cyanosis. Cyanosis is noted in approximately 50 percent of the newborn. According to Celermajer et al cyanosis for neonates is 74 percent. These patients are at risk for paradoxical emboli or cerebral abscess because of the interatrial communication (PFO or ASD).

Intense central cyanosis suggests restrictive ASD, while intense peripheral cyanosis with severe metabolic acidosis in the newborn indicates inadequate ductus arteriosus. Cyanosis is exaggerated in tricuspid valve stenosis or regurgitation. Eventually, left heart failure may develop due to prolonged cyanosis, interventricular dependence and left heart fibrosis.

Asymptomatic cyanotic newborn do not need any active treatment unless cyanosis is severe. Treatment consists of temporarily keeping the PDA open using prostaglandin (PG) E1 infusion $(0.05-0.1 \mathrm{mcg} / \mathrm{kg} / \mathrm{min})$ until PVR drops. Restrictive PFO/ASD may require balloon a trial septostomy in order to stabilize the baby for further management. While this may relieve systemic venous congestion, one should be aware of the increase in cyanosis that may occur due to increased right to left shunting at the atrial level after septostomy.

Indications for surgery in newborns and infants with cyanosis and congestive heart failure (CHF) are not clear and the decision to perform surgery should be individualized. Significant cyanosis $(80 \%$ or less; Hemoglobin 16 gm \% or more) in older child or adult is an indication for surgery.

Pregnancy is well tolerated if the patient does not have cyanosis, right heart failure or arrhythmias.

Cyanosis in pulmonary valve disease: In a neonate with severe valvular PS, the pulmonary flow may be completely duct dependent i.e. functional pulmonary atresia. Neonates with critical PS present with cyanosis, $\mathrm{CF}$, hypotension, feeding difficulty, tachypnea, without appreciable murmur. The affected neonate has cyanosis, congestive heart failure and hypotension. Any increase in oxygen demand, such as exercise, may result in frank peripheral cyanosis. Rarely, children with severe PS may maintain patency of the PFO allowing right-to-left shunt at times of peak exercise (exercise-induced cyanosis).

In patients with a patent foramen ovale or atrial septal defect, central cyanosis is observed as a result of rightto-left atrial shunting when the right atrial pressure exceeds the left atrial pressure. Progressive hypertrophy and decreased compliance of the right ventricle or myocardial failure with subsequent dilation may lead to central cyanosis in some patients.

\section{PS WITH INTERATRIAL COMMUNICATION:}

1. The exertional dyspnea generally precedes the onset of cyanosis and is out of proportion to the cyanosis.

2. Cyanosis of varying degree can present from birth to later in life.

a. Patients with critical PS present with cyanosis at birth.

b. In the TOF patients, the cyanosis and dyspnea are directly proportionate to each other and usually appear in early infancy.

c. In the degree of cyanosis, trilogy of Fallot patients occupy an intermediate place between TOF and Eisenmenger syndrome (In tetralogy cyanosis occurs 2-3 month after birth, all cyanotic by $5-8$ years, even on rest, in contrast in triology cyanosis usually in late childhood, puberty or adulthood, mild cyanosis appears on exertion initially).

Cyanosis in mitral atresia: Cyanosis is due to pulmonary stenosis or atresia. Adequacy of pulmonary blood flow determined by pulmonary outflow tract obstruction, size of patent ductus arteriosus, pulmonary vascular resistance and degree of restriction at the atrial level shunt. Causes of death included heart failure, cyanosis and septicemia.

Cyanosis in interruption of aortic arch: Differential cyanosis will be present. The differential cyanosis may 
or may not be clinically appreciated, better to do exercise to bring out.

In TGA as the pulmonary artery is connected to the left ventricle, it carries the more oxygenated blood. The lower half of the body will have higher oxygen saturation compared to the upper part of the body in a condition known as reversed differential cyanosis. In neonates with significant lung disease and pulmonary hypertension due to the pulmonary venous desaturation, reversed differential cyanosis may not be apparent. In the presence of an aberrant right subclavian artery arising after the interruption, the oxygen saturation measured on the right arm may not be different from those measured in the lower extremities. Cyanosis due to vascular sling: Cyanosis is seen due to Brachiocephalic Artery compression of Airways.

Cyanosis in TOF: The degree of right ventricular outflow tract obstruction (RVOT) often correlates with the degree of cyanosis. In less severe cases, cyanosis is first noticed during crying. Typical spell is characterized by progressive increase in the rate and depth of respiration, deepening cyanosis, limpness or syncope.

Cyanosis in TOF is determined by the severity of pulmonary stenosis and also to a lesser extent by systemic to pulmonary collaterals. In the case of pulmonary atresia, cyanosis sometimes may be absent due to systemic pulmonary collaterals.

Unoperated adults with tetralogy of Fallot (TOF) have high morbidity. They may present with progressive cyanosis, atrial arrhythmias, impaired biventricular function causing heart failure, paradoxical emboli through intracardiac shunts leading to thromboembolic events and cerebral abscesses. Progressive cyanosis and complications associated with prolonged cyanosis are associated with the long-term residua and sequelae after palliated shunts.

One of the causes of congestive heart failure on TOF post repair is prolonged cyanosis in patients with late repair along with long-standing shunts, poor myocardial preservation during multiple surgeries and right heart failure due to severe pulmonary regurgitation.

Cyanosis in pulmonary atresia with intact ventricular septum: Cyanosis is the most common presentation. The spectrum of presentation ranges from cyanosis evident after ductal narrowing/closure in a neonate to congestive cardiac failure in a pink child. Some children have balanced circulation and present late with cyanosis and exertional dyspnea.

\section{CYANOSIS IN DORV:}

1. VSD type of DORV - They may have mild cyanosis or no cyanosis at all.

2. TOF type of DORV - Cyanosis is present from early months of life; a systolic murmur is audible in the newborn period. These children may have progressively worsening cyanosis with cyanotic spells.

3. Remote VSD type of DORV: They present like patients with single ventricle. Children have mild cyanosis and the pulmonary blood flow may be balanced, increased or decreased.

CYANOSIS IN TRUNCUS: Truncus with Babies with interrupted aortic arch or coarctation of aorta may present precipitously when the ductus arteriosus closes in the neonatal period. Such patients may constitute approximately 10 percent of the patients with Truncus arteriosus. Manifestations would include circulatory collapse, metabolic acidosis, respiratory distress and cyanosis.

Mild cyanosis may be present from decreased pulmonary blood flow due to either pulmonary artery ostial stenosis or high pulmonary vascular resistance that is usual in neonatal period (Fig 1). Cyanosis resolves when the pulmonary vascular resistance decreases with transition to neonatal circulation. In older children, cyanosis develops again as Eisenmenger syndrome occurs with development of pulmonary vascular obstructive disease. If the pulmonary blood flow is normal or diminished due to anatomic pulmonary artery stenosis or persistence of high pulmonary vascular resistance, the child will present without features of heart failure or respiratory distress. There may be mild cyanosis, however 
Fig.1: Flow chart of approach in an infant with cyanosis. Courtesy of IB Vijayalaxmi et al [26]

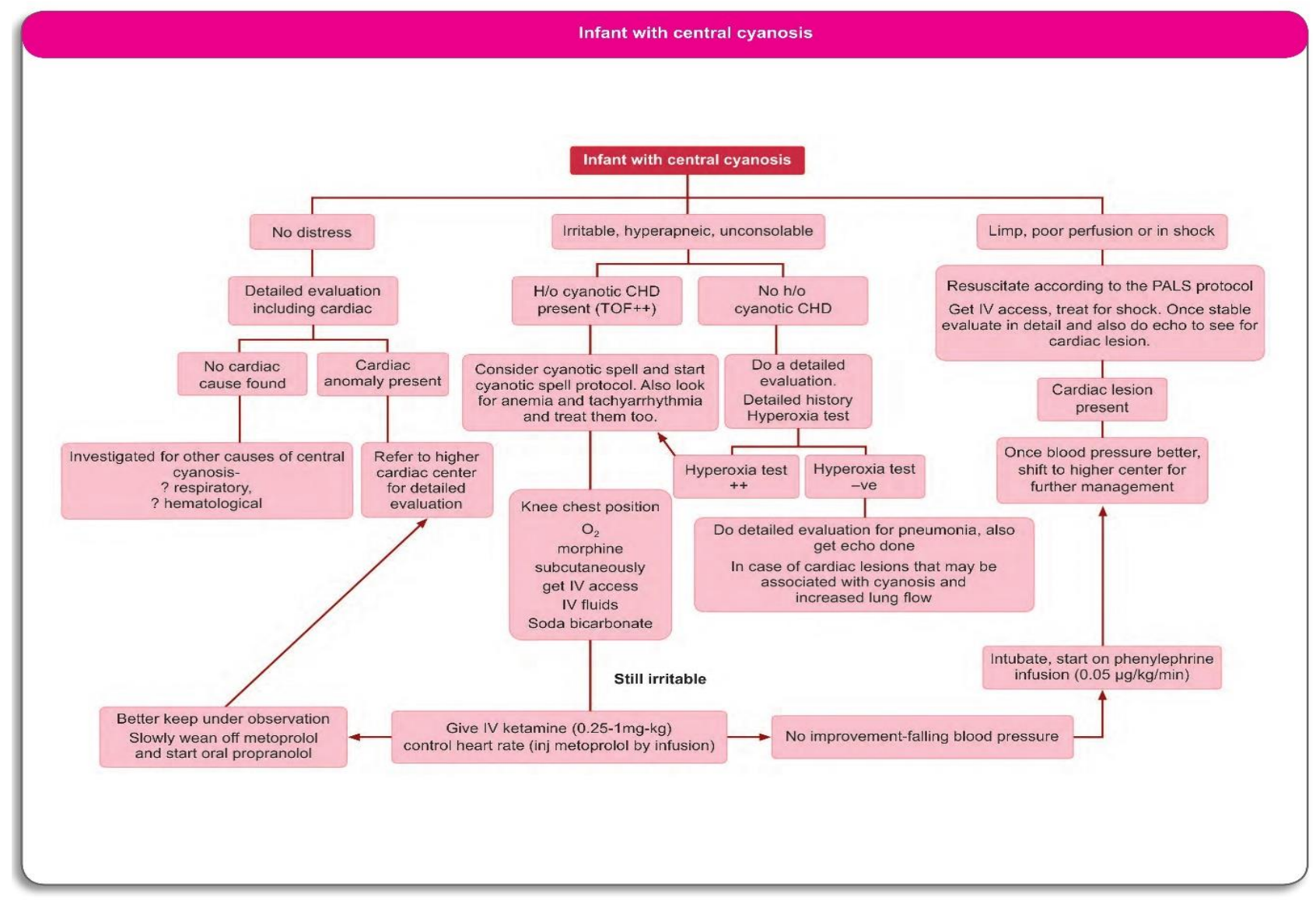

\section{CYANOSIS IN D- TRANSPOSITION OF GREAT ARTERIES:}

Simple transposition of great arteries usually presents in the neonatal age group with severe cyanosis. In a simple TGA, reverse differential cyanosis will be present: the postductal saturation will be higher than the preductal one. Presence of a VSD is not an insurance against cyanosis, as flow of blood across the VS will be predominantly unidirectional. Severe cyanosis accompanied by (paradoxically) increased pulmonary vascular markings on chest radiograph is a pointer to the diagnosis of DTGA. While a Rastelli repair in early infancy protects from prolonged cyanosis, the infantsized conduit needs more reoperations for replacement over a lifetime. Baffle leak after atrial switch operation Right to-left shunts are clinically more significant if they are contributing to worsening systemic arterial oxygenation and cyanosis.
Cyanosis in cTGA: Cyanosis may be seen in patients with significant left ventricular outflow tract (VT) obstruction associated with a VSD in cTGA.

CYANOSIS IN SINGLE VENTRICLE: The most frequent presenting symptom is cyanosis since birth. Cyanosis may not be very obvious if the patient has increased PBF. In neonates with single ventricle and subpulmonary stenosis/ atresia marked cyanosis, metabolic acidosis develops and they become critical as the ductus closes.

In a post bidirectional Glenn shunt child presenting with cyanosis, it may be due to nonfunctioning Glenn or significant descending venoenous collateral decompressing the Glenn shunt. In fenestration in the intra-atrial tunnel at the time of TCPC for patient with increased risk for surgery will have mild cyanosis.A considerable percentage of small fenestrations will close spontaneously later on. In patients with persistently patent fenestration and mild cyanosis it remains 
controversial whether the interventional occlusion is required later. In patients with increasing cyanosis during exercise, transcatheter device occlusion of fenestration is recommended in the presence of appropriate hemodynamic.

In fonton circulation, if cyanosis develops, careful angiographic assessment of the circulation is required. Right-to-left shunts through venous collaterals may be emblazed or intracardiac shunts closed. However, sealing the 'release valve' from the high-resistance pulmonary circulation may reduce cardiac output and cause systemic venous congestion.

Following fonton's, worsening cyanosis may be due to a) Existence of surgically created communication (fenestration), b) Development of collateral arteriovenous circulation (systemic and pulmonary). The development of pulmonary arteriovenous malformations is described in up to 25 percent of patients post-Glenn shunt leading to progressive cyanosis.

\section{CYANOSIS IN HYPOPLASTIC LEFT HEART} SYNDROME : As the ductus arteriosus begins to close (normally over the first 244 hours of life), symptoms of cyanosis, tachypnea, respiratory distress, pallor, lethargy, metabolic acidosis, and oliguria develop. Respiratory symptoms and profound cyanosis are apparent at birth in $25 \%$ of cases.

The possible reasons for cyanosis include:

a. Pulmonary venous desaturation from lung pathology (pneumothorax, pleural effusion, pneumonia, infection, pulmonary edema)

b. Systemic venous desaturation (anemia, low systemic cardiac output)

C. Decreased pulmonary blood flow (elevated PVR, pulmonary venous hypertension, restrictive the atrial septal defect, distortion of pulmonary arteries, small or occlusion of systemic to pulmonary artery shunt).

CYANOSIS IN ENDOCARDIAL FIBROELASTOSIS: There is no cyanosis, but peripheral cyanosis may be seen in severe cases of heart failure.

CYANOSIS IN PULMONARY ARTERIAL HYPERTENSION: These patients may have cyanosis which worsens with exercise. Cyanosis is a late finding and usually attributable to a markedly reduced cardiac output, with systemic vasoconstriction and ventilationperfusion mismatch in the lung and rarely due to the right to left shunting across the stretched patent foramen ovale. PAH associated with systemic to pulmonary shunts, no cyanosis is present at rest. In post-tricuspid shunts congestive cardiac failure CC occurs in infancy and cyanosis can occur later in the second decade, once goes into Eisenmenger stage (ES). In complex cyanotic CHD (single ventricle, truncus etc) the cyanosis is progressive throughout life. In ES, A right to left shunt at atrial level even augments left ventricular filling and may prevent heart failure during exercise, however, it is at the expense of cyanosis during exercise.

\section{CYANOSIS AND INFECTIVE ENDOCARDITIS:}

The presence of cyanosis in CHD adds tremendously to the overall risk of developing IE. Among unrepaired cardiac defects, the lifetime risk for IE in cyanotic CHD is up to 8.2 cases per 1,000 patients per year, while the lifetime risk for IE in a cyanotic CHD is 1.7 to 2.4 cases per 1,000 patients per year.

\section{INFANTS WITH HEART FAILURE + MILD CYANOSIS:}

Table 1: Infants with Heart failure and mild cyanosis

1. Transposition of great arteries with VSD

2. Double outlet right ventricle

3. Tricuspid atresia with large VSD (with or without transposition of great arteries)

4. Single ventricle without pulmonary stenosis

5. Total anomalous pulmonary venous return (TAPVR).

6. Truncus arteriosus

\section{CYANOSIS AND PRE-OP EVALUATION:}

Cyanosis and congestive heart failure (CHF) are two clinical states that can produce adverse pathophysiological consequences. For children with cyanotic lesions, questions should be directed at determining: 
1. Is the cyanosis static, progressive, intermittent, or continuous.

2. New onset of cyanotic spells?

3. Evaluation of arterial blood gas (ABG) along with serum lactate level should be reserved for the children with, suspected respiratory compromise or severe cyanosis.

\section{PRECAUTIONS TO BE TAKEN DURING SURGERY IN A CYANOTIC PATIENT:}

Inhalational anesthetic during surgery, because of their propensity to cause peripheral vasodilatation, may result in increased shunt and cyanosis in children with right-to-left shunts. Inhaled sevoflurane is generally well tolerated and is the preferred approach in children without intravenous access. Patients with reduced PBF have a delay in anesthetic uptake. In extreme cyanosis, sevoflurane, because of its reduced solubility, may not achieve an adequate alveolar concentration to fully induce anesthesia. Halothane, because of its greater solubility, would be more efficacious in extreme cyanosis.

\section{POSTOPERATIVE MANAGEMENT IN A CYANOTIC PATIENT:}

Aspirin for pain management - The risks may be greater in children than in adults because of the presence of collateral vessels, increased venous pressure, coagulopathy related to cyanosis, and the use of aspirin. In children with CHD, especially those with cyanosis or systemic hypoperfusion, coagulation factors often continue to be depressed secondary to impaired hepatic protein synthesis

\section{POSTSURGICAL CYANOSIS:}

Post TOF intracardiac repair with PA conduit: Presence of right to left shunting at the level of systemic veins to pulmonary veins, atrial baffle leaks, patent foramen ovale, and PA to pulmonary veins should be explored.

Pulmonary venous desaturation in the setting of pulmonary venous hypertension due to (a) systolic or diastolic subaortic ventricular failure, (b) AV valve regurgitation, (c) intravascular obstruction or extravascular compression by an enlarging RA or PA should be explored.

\section{CYANOSIS IN CATH LAB:}

Malignant hyperthermia can occur in the catheterization laboratory, particularly when halothane and/or succinylcholine are used in patients receiving general anesthesia. This is an autosomal dominant condition, which results in a rapid and marked rise in body temperature, tachycardia, tachypnea, respiratory and metabolic acidosis, cyanosis and eventually generalized muscle rigidity and myoglobinuria.

\section{Cyanosis is an indication for closure of PFO:}

In the following diseases, if cyanosis is there then PFO closure percutaneously is indicated.

1. Platypnea-orthodeoxia.

2. Following a pneumonectomy.

3. Right ventricular malfunction associated with congenital heart defects.

4. Right ventricular malfunction associated with chronic obstructive pulmonary disease

5. Right to left shunt following a right ventricular myocardial infarct.

\section{CYANOTIC FEMALE AND PREGNANCY:}

Siu et al described a risk index scoring system to predict the risk of adverse maternal events. In order to calculate the score, the risk index awards one point each for

- Poor functional status (NYHA FC > II),

- Cyanosis (oxygen saturation $<90 \%$ ),

- LVsystolic dysfunction,

- Left heart obstruction

- History of cardiac events prior to pregnancy including arrhythmias, stroke, or pulmonary edema.

Maternal cyanosis is a fetal risk factor for wastage (6$27 \%$ ), prematurity and dysmaturity. Occurrence of CHD in the offspring varies from 2 to 18 percent.

One of the highest thrombotic risk groups includes those women with $\mathrm{CHD}$ with cyanosis or fontan procedure and cautioned against the use of combined hormonal contraceptives.

Ebstein's female, in the absence of significant maternal cyanosis or arrhythmia, pregnancy is usually well tolerated 


\section{CYANOTIC PATIENT AND EXERCISE:}

The main determinants of exercise tolerance besides the severity of the underlying CHD lesions are ventricular function, pulmonary hypertension, cyanosis and arrhythmias. The cyanosis worsens with effort due to increasing hypoxemia (decrease in oxygen saturations measured by pulse oximetry). Even after palliative procedures that relieve the cyanosis at rest, exercise tolerance may still be depressed due to hypoxemia with moderate to heavy exercise.

\section{CHRONIC SEQUELAE OF CYANOSIS:}

1. Defective hemostasis-- abnormalities in platelet function, coagulation and fibrinolytic systems.

2. Secondary polycythemia and consequent complications.

3. Contrast-induced acute tubular necrosis and vascular thrombosis due to lessen glomerular filtration rate and increase viscosity.

4. The paucity of atherosclerosis - may be due to hypercholesterolemia that persists after surgical elimination of the cyanosis.

5. Cholelithiasis and asymptomatic gallstonesprevalence are significantly high in cyanotic CHD patients regardless of cardiac repairs. In addition to prolonged cyanosis in these patients, exposure to frequent cardiopulmonary bypass during surgeries and thrombocytopenia might influence gallstone formation in adults with CHD.

6. In patients with cyanotic conditions, the long-term compensation for chronic cyanosis shows a major redistribution of organ perfusion with selected blood flow to the heart, brain, lung, and kidney and decreased flow to the splanchnic circulation, skin, muscle, and bone.

7. Chronic cyanosis is associated with increased work of breathing in an attempt to increase oxygen uptake and delivery.

8. Often, patients whose pulmonary blood flow is dependent entirely on a surgically created systemic to pulmonary artery shunt or on one or more native, systemic to pulmonary artery collateral arteries will develop increasing cyanosis over time or occasionally acutely clot or otherwise close off.
COMBINED CYANOSIS: In cardiogenic shock with pulmonary edema there may be a mixture of both types.

\section{DIFFERENTIAL CYANOSIS:}

Patient will have cyanosis that is more intense in the feet than in the hands. With pulse oximetry, if the pre-ductal saturation is higher than the postductal saturation (3 to 7 $\%$ difference), differential cyanosis exists. This differential cyanosis is seen in left heart obstructive lesions such as Coarctation of Aorta, interrupted aortic arch, hypoplastic left heart syndrome, critical aortic stenosis or pulmonary hypertension with PDA. This provides information about the structural abnormality and anatomy.

Table 7: PDA with differential cyanosis - clinical implications.

\begin{tabular}{|l|l|}
\hline Clinical finding & Diagnosis \\
\hline $\begin{array}{l}\text { Cyanosis and clubbing of } \\
\text { the toes associated with } \\
\text { pink fingernails of the } \\
\text { right hand and cyanosis } \\
\text { and clubbing of the left } \\
\text { hand }\end{array}$ & $\begin{array}{l}\text { PDA with normally related great vessels } \\
\text { and a reversed shunt as a result of } \\
\text { pulmonary hypertension, with the patent } \\
\text { ductus arteriosus delivering cyanotic } \\
\text { blood to the left arm and lower } \\
\text { extremities. } \\
\text { Interruption of the aortic arch and a PDA } \\
\text { delivering desaturated blood to the } \\
\text { legs.If the right subclavian artery arises } \\
\text { proximal to the aortic obstruction, the } \\
\text { right hand can be pink and the left-hand } \\
\text { cyanoticWhen an anomalous right } \\
\text { subclavian artery originates from the } \\
\text { descending aorta, both hands are } \\
\text { cyanotic }\end{array}$ \\
\hline $\begin{array}{l}\text { Complete transposition of the great } \\
\text { vessels with preductal coarctation or } \\
\text { complete interruption of the aortic arch, } \\
\text { pulmonary hypertension, and a reverse } \\
\text { greater than in the toes } \\
\text { shunt through a patent Ductus arteriosus, } \\
\text { in this case delivering oxygenated blood } \\
\text { to the lower extremities }\end{array}$ \\
\hline
\end{tabular}

In TGA as the pulmonary artery is connected to the left ventricle, it carries the more oxygenated blood. The lower half of the body will have higher oxygen saturation compared to the upper part of the body in a condition known as reversed differential cyanosis. 


\section{HISTORY AND SYMPTOMS IN A CASE OF CYANOSIS:}

- Age of onset:

- Nature of onset:

a. Acute onset of cyanosis - pulmonary emboli, cardiac failure, pneumonia or asthma.

b. Chronic- COPD, associated polycythemia may exacerbate the degree of cyanosis.

c. Intermittent- The description may be typical of Raynaud's phenomenon.

- Past history: cyanosis can result from any lung disease of sufficient severity.

- Drug history: certain drugs may cause methemoglobinemia (E.g nitrates, dapsone) or sulfhaemoglobinaemia (E.g metoclopramide).

- Associated symptoms:

a. Chest pain: pleuritic chest pains may be due to pulmonary emboli or pneumonia. Pulmonary oedema may cause dull, aching chest tightness.

b. Dyspnoea: sudden onset of dyspnoea can occur with pulmonary emboli, pulmonary oedema or asthma.

\section{Clinical examination:}

- Temperature: infection.

- Inspection:

a. Central cyanosis produces a blue discoloration of the mucous membranes of the lips and tongue as well as the extremities.

b. Peripheral cyanosis affects the extremities and the skin around the lips but not the mucous membranes.

c. The combination of clubbing and cyanosis is frequent in congenital heart disease and may also occur in pulmonary disease (lung abscess, bronchiectasis, cystic fibrosis) and pulmonary arteriovenous shunts.

d. The jugular venous pressure is elevated with congestive cardiac failure.

- Respiratory examination:

a. Poor chest expansion occurs with chronic bronchitis and asthma. Unilateral reduced chest expansion may occur with lobar pneumonia.

b. Dullness to percussion occurs over an area of consolidation. c. Localized crepitation may be heard with lobar pneumonia. Crepitation is more widespread with bronchopneumonia and pulmonary oedema. Air entry may be poor with COPD and asthma. Bronchial breathing may be auscultated over an area of consolidation, and wheezing may be heard with asthma.

- Cardiac examination:

a. Heart sounds may be abnormal or added heart murmurs may suggest a cardiac origin (more description will be given when individual diseases are dealt).

b. Heterotaxy syndrome(HS) - Clinical signs may include: abnormal heart rate and rhythm, low volume or differential pulses, abnormal or differential blood pressure in all four limbs, cyanosis, abnormal cardiac apex, with or without cardiomegaly, single second heart sound, with or without ejection or pansystolic or continuous murmur. In older children with isomerism of left atrial appendage (ILAA), second sound may be wide, variable or fixed.

- Localized features suggesting an aetiology of peripheral cyanosis, such as oedema in venous insufficiency or absent peripheral pulses and ischemia in arterial occlusion.

\section{INVESTIGATIONS:}

- Arterial blood gasses: oxygen saturation for patients with central cyanosis is usually below $85 \%$. If the oxygen saturation does not increase to above 95\% while the patient inhales $100 \%$ oxygen then there is likely to be pulmonary intravascular shunting of blood bypassing the alveoli (E.g right-to-left intracardiac shunt or pulmonary arteriovenous fistulae).

- FBC: hemoglobin level is increased with chronic cyanosis. White cell count is increased in pneumonia and pulmonary embolism.

- ECG: features of myocardial infarction; nonspecific ST abnormalities with pulmonary emboli.

- CXR: pneumonia, pulmonary infarction, cardiac failure.

- $\quad$ Sputum and blood cultures: pneumonia. 
- Ventilation-perfusion scan - 'VQ scan', or pulmonary angiography: pulmonary embolus.

- Echocardiography: cardiac defects.

- Haemoglobin spectroscopy: methemo globinaemia, sulfhae moglobinaemia.

- Digital subtraction angiography: acute arterial occlusion.

- Duplex Doppler or venography: acute venous occlusion.

\section{MANAGEMENT}

- Oxygen therapy for patients who are hypoxic.

- Treatment of the underlying cause.

- Management of cyanotic spell- follow the protocol. Sedation, morphine, beta-blocker (esmolol, metoprolol) infusion, IV fluid, soda bicarb and oxygen, blood transfusion or Ventilation if needed. Refer for surgery or stenting, BAS.

\section{REFERENCES:}

1. Lundsgaard C, Van Slyke DD. Cyanosis. Medicine. 2(1):1-76.

2. Mini Oxford Handbook of Clinical Medicine (7th ed.). p. 56.

3. Cyanosis. Lunds gaard C, Van SD, Abbott ME. Cyanosis. Can Med Assoc J 1923 Aug;13 (8):601-

4. Goss GA, Hayes JA, Burdon JG. Deoxyhemoglobin concentrations in the detection of central cyanosis. Thorax 1988 Mar; 43(3):212-13.

5. Mosby's Medical, Nursing \& Allied Health Dictionary. Mosby-Year Book (4th ed.). 1994. p. 425.

6. Jump up^ Met hemoglobin.

7. Das S, Maiti A; Acrocyanosis: an overview. Indian J Dermatol. 2013 Nov; 58(6):417-20. doi: 10.4103/00195154.119946.

8. Fernandez-Frackelton M. Cyanosis. In: Marx JA, Hockberger RS, Walls RM, et al, eds. Rosen's Emergency Medicine: Concepts and Clinical Practice. 8th ed. Philadelphia, PA: Elsevier Mosby; 2014: chap 14.

9. Clinical Methods: The History, Physical, and Laboratory Examinations. 3rd edition. Walker HK, Hall WD, Hurst JW, editors. Boston: Butterworths; 1990. Chapter 45. Cyanosis.

10. Blount SG Jr. Cyanosis: pathophysiology and differential diagnosis. Prog Cardiovasc Dis. 1971;13:595-605.

11. Comroe JH Jr,, Botelho AB. The unreliablity of cyanosis in the recognition of arterial anoxemia. Am J Med Sci.1947; 214:1-6.

12. Edwards EA, Duntley SQ. The pigments and color of living human skin. Am J Anat. 1939;65:1-33.

13. Finch CA. Methemoglobinemia and sulfhemoglobinemia. N Engl J Med. 1948; 239:470-78.

14. Geraci JE, Wood EH. The relationship of the arterial oxygen saturation to cyanosis. Med Clin North Am.1951; 35:1185-1202.

15. Jeghers H. Pigmentation of the skin. N Engl J Med. 1944; 231:88-100. , 122-36,181-89.

16. Lundsgaard C, Van Slyke DD. Cyanosis. Medicine. 1923;2:1-76.

17. Mansouri A. Review: methemoglobinemia. Am J Med Sci. 1985;289:200-91.

18. Medd WE, French EB, Wyllie VMcA. Cyanosis as a guide to arterial oxygen desaturation. Thorax. 1959;14:247-50.

19. Stadie WC. The oxygen of the arterial and venous blood in pneumonia and its relationship to cyanosis. J Exp Med. 1919;30:215-43.

20. Lees MH. Cyanosis of the newborn infant. Recognition and clinical evaluation. J Pediatr. 1970; 77:484. 
21. ood . Attack $f$ eeper Cyanosis And oss $f$ Consciousness (Syncope) In Fallots Tetralogy. $r$ eartournal 15;20226.

22. De Castro CM, Nelson WP, Jones RC, et al. Pulmonary stenosis: cyanosis, interatrial communication and inadequate right ventricular distensibility following pulmonary valvotomy. Am J Cardiol. 1970;26:540-3.

23. Petit CJ, Gillespie MJ, Kreutzer J, et al. Endovascular stents for relief of cyanosis in singleventricle patients with shunt or conduit-dependent pulmonary blood flow. Catheter Cardiovasc Interv. 2006;68:280-86.

24. Perloff JK. Systemic complications of cyanosis in adults with congenital heart disease. Hematologic derangements, renal function and urate metabolism. Cardiol Clin. 1993;11:689-99.
25. A Comprehensive Approach to CONGENITAL HEART DISEASES text book by Dr. IB Vijayalaxmi, First Edition 2013.

26. Cardiac Catheterization in Congenital Heart Disease: Pediatric and Adult by Charles E. Mullins, First Edition 2006.

27. Cardiac catheterization, angiography and intervention. Grossman \&Baims, Eight edition.

28. DiSesaVJ , Cohn LH, Grossman W. Management of adults with congenital bidirectional shunts, cyanosis, and pulmonary vascular obstruction: successful operative repair in 3 patients. Am] Cardiol 1983;51:1495.

29. Harrison's principles of internal medicine, Edition.

30. Oxford case histories. 